\section{$\S 24$. Microwave Imaging Reflectometry in LHD}

Yamaguchi, S., Nagayama, Y., Pavlichenko, R., Inagaki, S., Kogi, Y., Mase, A. (KASTEC, Kyushu Univ.)

The microwave reflectometry is a radar technique for the measurement of the electron density profiles and fluctuations by probing the density-dependent cutoff layer in the plasma. A multi-channel reflectometry system equipped with an imaging optical system has been developed to the microwave imaging reflectometry (MIR) $[1$, 2]. It has a potential to obtain the 2-D/3-D image of the MHD turbulences and instabilities with excellent time and spatial resolutions. The MIR system is under development in the Large Helical Device (LHD).

Figure 1(a) shows the schematic of the MIR system of LHD. The illumination beams of 66 and $69 \mathrm{GHz}$ are launched from the port 4-O to the plasma center, and they are reflected at the right-hand cutoff layer in the peripheral plasma. Since the cutoff layer of X-mode is less bent and the wavelength of $\mathrm{X}$-mode is shorter than O-mode, the $\mathrm{X}$-mode illumination is utilized in LHD. The reflected beams are focused by the imaging optical system, and the reflected power is measured by the 6-channels heterodyne receiver. The dichroic plate has a clear cut-off feature at 70 $\mathrm{GHz}$, so that it can separate the operational frequency band for MIR (50-70 GHz) and ECE Imaging (ECEI) system $(70-140 \mathrm{GHz})[1,3]$.

The LHD plasma has an elliptical cross-section which is tilted at the port 4-O as shown in Fig.1(a). As the density is higher, the right-hand cutoff layer is more tilted downward near the equatorial plane. The beam paths near the reflection surface is calculated by using a ray-tracing code. It shows that the illumination beam should be launched upward by a few degree in order to obtain considerable reflected power. In the new setup an ellipsoidal mirror with adjustable angle is installed into the vacuum chamber of LHD. It can be rotated poloidally and toroidally by remote-controlled actuators with ultrasonic motors to optimize the illumination angle for the wider range of the plasma parameters.

Figure 1(b) shows the example of the MIR signals in the case of the low density plasma ( $R_{\mathrm{ax}}=3.5 \mathrm{~m}, B_{\mathrm{ax}}=-2.829 \mathrm{~T}$, $\gamma=1.254$ and $B_{\mathrm{q}}=100 \%$ ). A slow oscillation with large amplitude appears in the MIR signal right after the ECRH is turned off at $\mathrm{t}=0.46 \mathrm{sec}$. The frequency varies from 20 to $40 \mathrm{~Hz}$ during $\mathrm{t}=0.5-1.0 \mathrm{sec}$. Since the oscillation signals are slightly different among the different channels, the oscillation is not a mechanical vibration. The amplitude of the oscillation is reduced and the oscillation disappears after the density is increased at $t=1.0 \mathrm{sec}$. The corresponding oscillation is not observed in the ECE signals, therefore, the slow oscillation may be a density fluctuation in the plasma. This indicates that the MIR has a potential to observe phenomena that is not observed by ECE measurement.

\section{Reference}

1) Nagayama, Y. et al. : J. Plasma Fusion Res. 81 (2005) 337

2) Mazzucato, E. et. al. : Nuclear Fusion 41 (2001) 203

3) Mase, A. et al. : Rev. Sci. Instrum. 74 (2003) 1445
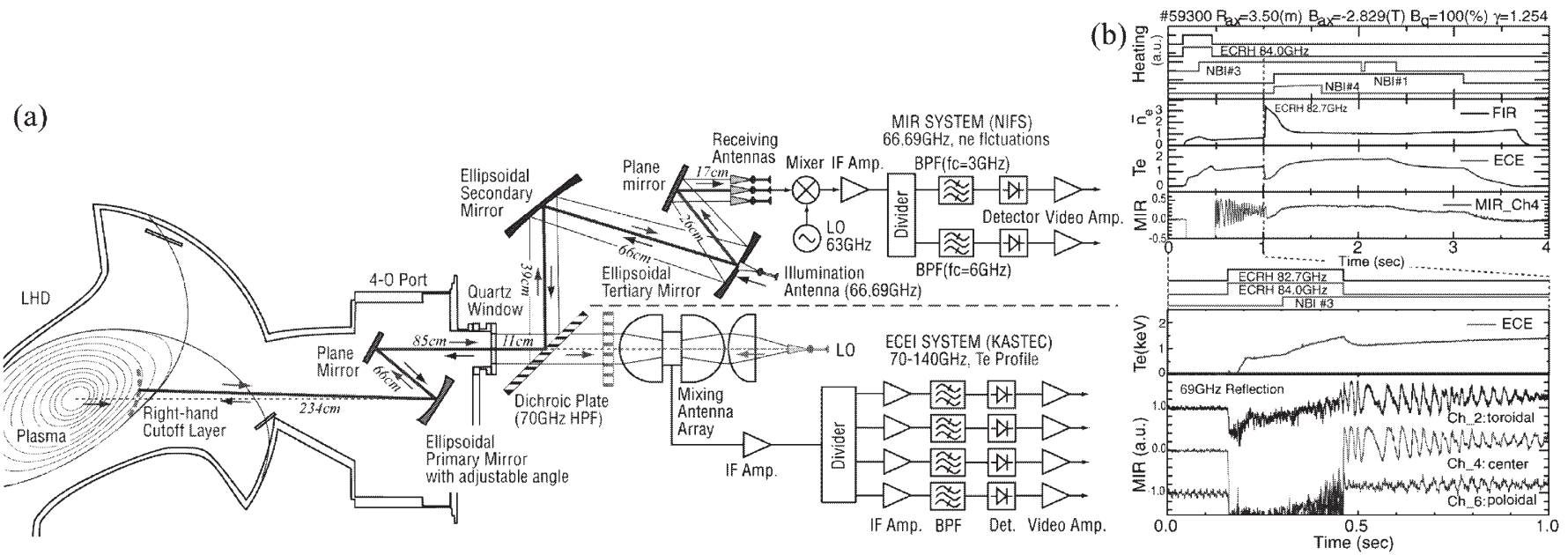

Fig.1. Schematic of the MIR system (a) and oscillations of the MIR signals in the case of the low density plasma (b). 